\title{
On the Bicomplex $k$-Fibonacci Quaternions
}

\author{
Fügen Torunbalcı Aydın ${ }^{1 *}$
}

\begin{abstract}
In this paper, bicomplex $k$-Fibonacci quaternions are defined. Also, some algebraic properties of bicomplex $k$-Fibonacci quaternions are investigated. For example, the summation formula, generating functions, Binet's formula, the Honsberger identity, the d'Ocagne's identity, Cassini's identity, Catalan's identity for these quaternions are given. In the last part, a different way to find $n-t h$ term of the bicomplex $k$-Fibonacci quaternion sequence was given using the determinant of a tridiagonal matrix.
\end{abstract}

Keywords: Bicomplex Fibonacci quaternion, Bicomplex $k$-Fibonacci quaternion, Bicomplex number, $k$-Fibonacci number, Tridiagonal matrix

2010 AMS: Primary 11R52, 11B39, 20G20

${ }^{1}$ Yildiz Technical University Faculty of Chemical and Metallurgical Engineering Department of Mathematical Engineering Davutpasa Campus, 34220 Esenler, Istanbul, TURKEY

*Corresponding author: ftorunay@gmail.com; faydin@yildiz.edu.tr

Received: 21 March 2019, Accepted: 2 August 2019, Available online: 30 September 2019

\section{Introduction}

In 2007, the $k$-Fibonacci sequence $\left\{F_{k, n}\right\}_{n \in \mathbb{N}}$ is defined by Falcon and Plaza [1,2] as follows

$$
\left\{\begin{aligned}
F_{k, 0}= & 0, F_{k, 1}=1 \\
F_{k, n+1}= & k F_{k, n}+F_{k, n-1}, n \geq 1 \\
\text { or } & \\
\left\{F_{k, n}\right\}_{n \in \mathbb{N}}= & \left\{0,1, k, k^{2}+1, k^{3}+2 k, k^{4}+3 k^{2}+1, \ldots\right\} .
\end{aligned}\right\}
$$

Here, $k$ is a positive real number.

In 2015, Ramirez [3] defined the the $k$-Fibonacci and the $k$-Lucas quaternions as follows:

$$
D_{k, n}=\left\{F_{k, n}+\mathbf{i} F_{k, n+1}+\mathbf{j} F_{k, n+2}+\mathbf{k} F_{k, n+3} \mid F_{k, n}, n-\text { th } k \text {-Fibonacci number }\right\},
$$

and

$$
P_{k, n}=\left\{L_{k, n}+\mathbf{i} L_{k, n+1}+\mathbf{j} L_{k, n+2}+\mathbf{k} L_{k, n+3} \mid L_{k, n}, n-\text { th } k \text {-Lucas number }\right\}
$$

where $\mathbf{i}, \mathbf{j}$ and $\mathbf{k}$ satisfy the multiplication rules

$$
\mathbf{i}^{2}=\mathbf{j}^{2}=\mathbf{k}^{2}=-1, \mathbf{i} \mathbf{j}=-\mathbf{j} \mathbf{i}=\mathbf{k}, \quad \mathbf{j} \mathbf{k}=-\mathbf{k} \mathbf{j}=\mathbf{i}, \quad \mathbf{k} \mathbf{i}=-\mathbf{i} \mathbf{k}=\mathbf{j} .
$$

In 1892, bicomplex numbers were introduced by Corrado Segre, for the first time [4]. In 1991, G. Baley Price, the bicomplex numbers gave in the book based on multicomplex spaces and functions [5]. The set of bicomplex numbers can be expressed by a basis $\{1, i, j, i j\}$ as,

$$
\mathbb{B C}=\left\{q=q_{1}+i q_{2}+j q_{3}+i j q_{4} \mid q_{1}, q_{2}, q_{3}, q_{4} \in \mathbb{R}\right\}
$$


where $i, j$ and $i j$ satisfy the conditions

$$
i^{2}=-1, j^{2}=-1, i j=j i \text {. }
$$

In 2019, bicomplex $k$-pell quaternions were introduced by Catarino Paula, [6] as follows

$$
\mathbb{B C}_{k, n}^{P}=\left\{P_{k, n}+i P_{k, n+1}+j P_{k, n+2}+i j P_{k, n+3} \mid P_{k, n}, n-t h k \text {-Pell number }\right\},
$$

where $i, j$ and $i j$ satisfy the conditions

$$
i^{2}=-1, j^{2}=-1, i j=j i \text {. }
$$

The aim of this study is to define bicomplex $k$-Fibonacci quaternions with $k$-Fibonacci number and bicomplex number and to give their algebraic properties.

\section{The bicomplex $k$-Fibonacci numbers}

Definition 2.1. The bicomplex $k$-Fibonacci and $k$-Lucas numbers can be define by with the basis $\{1, i, j, i j\}$, where $i, j$ and $i j$ satisfy the conditions

$$
i^{2}=-1, j^{2}=-1, i j=j i,(i j)^{2}=1 .
$$

as

$$
\begin{aligned}
\mathbb{B C} F_{k, n} & =\left(F_{k, n}+i F_{k, n+1}\right)+j\left(F_{k, n+2}+i F_{k, n+3}\right) \\
& =F_{k, n}+i F_{k, n+1}+j F_{k, n+2}+i j F_{k, n+3}
\end{aligned}
$$

and

$$
\begin{aligned}
\mathbb{B} C L_{k, n} & =\left(L_{k, n}+i L_{k, n+1}\right)+j\left(L_{k, n+2}+i L_{k, n+3}\right) \\
& =L_{k, n}+i L_{k, n+1}+j L_{k, n+2}+i j L_{k, n+3} .
\end{aligned}
$$

For two bicomplex $k$-Fibonacci numbers, addition and subtraction are defined by the following:

$$
\mathbb{B C} F_{k, n} \pm \mathbb{B} C F_{k, m}=\left(F_{k, n} \pm F_{k, m}\right)+i\left(F_{k, n+1} \pm F_{k, m+1}\right)+j\left(F_{k, n+2} \pm F_{k, m+2}\right)+i j\left(F_{k, n+3} \pm F_{k, m+3}\right)
$$

and multiplication of by

$$
\begin{aligned}
\mathbb{B C} F_{k, n} \times \mathbb{B} C F_{k, m}= & \left(F_{k, n} F_{k, m}-F_{k, n+1} F_{k, m+1}-F_{k, n+2} F_{k, m+2}-F_{k, n+3} F_{k, m+3}\right) \\
& +i\left(F_{k, n} F_{k, m+1}+F_{k, n+1} F_{k, m}-F_{k, n+2} F_{k, m+3}-F_{k, n+3} F_{k, m+2}\right) \\
& +j\left(F_{k, n} F_{k, m+2}+F_{k, n+2} F_{k, m}-F_{k, n+1} F_{k, m+3}-F_{k, n+3} F_{k, m+1}\right) \\
& +i j\left(F_{k, n} F_{k, m+3}+F_{k, n+3} F_{k, m}+F_{k, n+1} F_{k, m+2}+F_{k, n+2} F_{k, m+1}\right) \\
= & \mathbb{B C} F_{k, m} \times \mathbb{B} \mathbb{C} F_{k, n} .
\end{aligned}
$$

\section{The bicomplex $k$-Fibonacci quaternions and some basic properties}

In 2018, the bicomplex Fibonacci quaternions defined by Aydın Torunbalc1 [7] as follows

$$
Q_{F n}=F_{n}+i F_{n+1}+j F_{n+2}+i j F_{n+3}
$$

where quaternionic units satisfy the rules Eq. 1.1. In this section, firstly the bicomplex $k$-Fibonacci quaternions will be defined.

Definition 3.1. The bicomplex $k$-Fibonacci quaternions are defined by using the bicomplex numbers and $k$-Fibonacci numbers as follows

$$
\mathbb{B C}^{F_{k, n}}=F_{k, n}+i F_{k, n+1}+j F_{k, n+2}+i j F_{k, n+3}
$$

where quaternionic units satisfy the rules Eq. 1.1. 
Let $\mathbb{B} \mathbb{C}^{F_{k, n}}$ and $\mathbb{B} \mathbb{C}^{F_{k, m}}$ be two bicomplex $k$-Fibonacci quaternions. For two bicomplex $k$-Fibonacci quaternions, addition and subtraction are defined in the obvious way,

$$
\begin{aligned}
\mathbb{B C}^{F_{k, n}} \pm \mathbb{B C}^{F_{k, m}} & =\left(F_{k, n}+i F_{k, n+1}+j F_{k, n+2}+i j F_{k, n+3}\right) \pm\left(F_{k, m}+i F_{k, m+1}+j F_{k, m+2}+i j F_{k, m+3}\right) \\
& =\left(F_{k, n} \pm F_{k, m}\right)+i\left(F_{k, n+1} \pm F_{k, m+1}\right)+j\left(F_{k, n+2} \pm F_{k, m+2}\right)+i j\left(F_{k, n+3} \pm F_{k, m+3}\right) .
\end{aligned}
$$

and multiplication by

$$
\begin{aligned}
& \mathbb{B C}^{F_{k, n}} \times \mathbb{B C}^{F_{k, m}}=\left[F_{k, n} F_{k, m}-F_{k, n+1} F_{k, m+1}-F_{k, n+2} F_{k, m+2}+F_{k, n+3} F_{k, m+3}\right] \\
& +i\left[F_{k, n} F_{k, m+1}+F_{k, n+1} F_{k, m}-F_{k, n+2} F_{k, m+3}-F_{k, n+3} F_{k, m+2}\right] \\
& +j\left[F_{k, n} F_{k, m+2}-F_{k, n+1} F_{k, m+3}+F_{k, n+2} F_{k, m}-F_{k, n+3} F_{k, m+1}\right] \\
& +i j\left[F_{k, n} F_{k, m+3}+F_{k, n+1} F_{k, m+2}+F_{k, n+2} F_{k, m+1}+F_{k, n+3} F_{k, m}\right] \\
& =\mathbb{B C}^{F_{k, m}} \times \mathbb{B C}^{F_{k, n}} \text {. }
\end{aligned}
$$

The different conjugations for bicomplex $k$-Fibonacci quaternions are presented as follows:

$$
\begin{aligned}
& \left(\mathbb{B C}^{F_{k, n}}\right)^{*_{1}}=F_{k, n}-i F_{k, n+1}+j F_{k, n+2}-i j F_{k, n+3}, \\
& \left(\mathbb{B}^{F_{k, n}}\right)^{*_{2}}=F_{k, n}+i F_{k, n+1}-j F_{k, n+2}-i j F_{k, n+3}, \\
& \left(\mathbb{B}^{F_{k, n}}\right)^{*_{3}}=F_{k, n}-i F_{k, n+1}-j F_{k, n+2}+i j F_{k, n+3} .
\end{aligned}
$$

Therefore, the norm of the bicomplex $k$-Fibonacci quaternion $\mathbb{B C}^{F_{k, n}}$ is defined in three different ways as follows

$$
\begin{aligned}
& N\left(\mathbb{B C}^{F_{k, n}}\right)^{*_{1}}=\| \mathbb{B C}^{F_{k, n}} \times\left(\mathbb{B} \mathbb{C}^{\left.F_{k, n}\right)^{*} \|^{2}}\right. \\
& =\left|\left(F_{k, n}^{2}+F_{k, n+1}^{2}\right)-\left(F_{k, n+2}^{2}+F_{k, n+3}^{2}\right)+2 j\left(F_{k, n} F_{k, n+2}+F_{k, n+1} F_{k, n+3}\right)\right| \\
& =\left|F_{k, 2 n+1}-F_{k, 2 n+5}+2 j F_{k, 2 n+3}\right|=\mathbb{B C}^{F_{k, n}}\left(\mathbb{B C}^{F_{k, n}}\right)^{* 1} \text {, } \\
& \left.N_{(} \mathbb{B C}^{F_{k, n}}\right)^{*_{2}}=\left\|\mathbb{B C}^{F_{k, n}} \times\left(\mathbb{B C}^{F_{k, n}}\right)^{*_{2}}\right\|^{2} \\
& =\left|\left(F_{k, n}^{2}-F_{k, n+1}^{2}\right)+\left(F_{k, n+2}^{2}-F_{k, n+3}^{2}\right)+2 i F_{k, n} F_{k, n+1}+k F_{k, 2 n+3}\right|=\mathbb{B C}^{F_{k, n}}\left(\mathbb{B C}^{F_{k, n}}\right)^{*_{2}} \text {, } \\
& N_{(} \mathbb{B C}^{\left.F_{k, n}\right)^{*_{3}}}=\| \mathbb{B C}^{F_{k, n}} \times\left(\mathbb{B C}^{\left.F_{k, n}\right)^{*} \|^{2}}\right. \\
& =\left|\left(F_{k, n}^{2}+F_{k, n+1}^{2}\right)+\left(F_{k, n+2}^{2}+F_{k, n+3}^{2}\right)+2 i j\left(F_{k, n} F_{k, n+3}-F_{k, n+1} F_{k, n+2}\right)\right| \\
& =\left|F_{k, 2 n+1}+F_{k, 2 n+5}+2 i j(-1)^{n+1} k\right|=\mathbb{B C}^{F_{k, n}}\left(\mathbb{B C}^{F_{k, n}}\right)^{* 3} \text {. }
\end{aligned}
$$

In the following theorem, some properties related to the bicomplex $k$-Fibonacci quaternions are given.

Theorem 3.2. Let $\mathbb{B C}^{F_{k, n}}$ be the bicomplex $k$-Fibonacci quaternion. In this case, we can give the following relations:

$$
\begin{aligned}
& \mathbb{B} \mathbb{C}^{F_{k, n+2}}=\mathbb{B C}^{F_{k, n}}+k \mathbb{B C}^{F_{k, n+1}}, \\
& \left(\mathbb{B C}^{F_{k, n}}\right)^{2}+\left(\mathbb{B C}^{F_{k, n+1}}\right)^{2}=\quad \mathbb{B C}^{F_{k, 2 n+1}}+\left(k F_{k, 2 n+6}-F_{k, 2 n+3}\right)+i\left(F_{k, 2 n+2}-2 F_{k, 2 n+6}\right) \\
& +j\left(F_{k, 2 n+3}-2 F_{k, 2 n+5}\right)+i j\left(3 F_{k, 2 n+4}\right) \text {, } \\
& \left(\mathbb{B} \mathbb{C}^{F_{k, n+1}}\right)^{2}-\left(\mathbb{B} \mathbb{C}^{F_{k, n-1}}\right)^{2}=k\left[\mathbb{B} \mathbb{C}^{F_{k, 2 n}}-F_{k, 2 n+2}+k F_{k, 2 n+5}+i\left(F_{k, 2 n+1}-2 F_{k, 2 n+5}\right)\right. \\
& \left.+j\left(-F_{k, 2 n+2}-2 k F_{k, 2 n+3}\right)+i j\left(3 F_{k, 2 n+3}\right)\right] \text {, } \\
& \mathbb{B C}^{F_{k, n+1}}+\mathbb{B C}^{F_{k, n-1}}=\mathbb{B C}^{L_{k, n}} \\
& \mathbb{B C}^{F_{k, n+2}-} \mathbb{B C}^{F_{k, n-2}}=k \mathbb{B}^{L_{k, n}}
\end{aligned}
$$


Proof. (3.2): By the Eq.(3.1) we get,

$$
\begin{aligned}
\mathbb{B C}^{F_{k, n}}+k \mathbb{B C}^{F_{k, n+1}} & =\left(F_{k, n}+k F_{k, n+1}\right)+i\left(F_{k, n+1}+k F_{k, n+2}\right)+j\left(F_{k, n+2}+k F_{k, n+3}\right)+i j\left(F_{k, n+3}+k F_{k, n+4}\right) \\
& =F_{k, n+2}+i F_{k, n+3}+j F_{k, n+4}+i j F_{k, n+5} \\
& =\mathbb{B C}^{F_{k, n+2}} .
\end{aligned}
$$

(3.3): By the Eq.(3.1) we get,

$$
\begin{aligned}
\left(\mathbb{B C}^{F_{k, n}}\right)^{2}+\left(\mathbb{B} \mathbb{C}^{\left.F_{k, n+1}\right)^{2}=}\right. & \left(F_{k, 2 n+1}-F_{k, 2 n+3}-F_{k, 2 n+5}+F_{k, 2 n+7}\right)+2 i\left(F_{k, 2 n+2}-F_{k, 2 n+6}\right) \\
& +2 j\left(F_{k, 2 n+3}-F_{k, 2 n+5}\right)+2 i j\left(2 F_{k, 2 n+4}\right) \\
= & \left(F_{k, 2 n+1}+i F_{k, 2 n+2}+j F_{k, 2 n+3}+i j F_{k, 2 n+4}\right)-F_{k, 2 n+3}-F_{k, 2 n+5}+F_{k, 2 n+7} \\
& +i\left(F_{k, 2 n+2}-2 F_{k, 2 n+6}\right)+j\left(F_{k, 2 n+3}-2 F_{k, 2 n+5}\right)+i j\left(3 F_{k, 2 n+4}\right) \\
= & \mathbb{B} \mathbb{C}_{k, 2 n+1}+\left(k F_{k, 2 n+6}-F_{k, 2 n+3}\right) \\
& +i\left(F_{k, 2 n+2}-2 F_{k, 2 n+6}\right)+j\left(F_{k, 2 n+3}-2 F_{k, 2 n+5}\right)+i j\left(3 F_{k, 2 n+4}\right) .
\end{aligned}
$$

(3.4): By the Eq.(3.1) we get,

$$
\begin{aligned}
& \left(\mathbb{B C}^{F_{k, n+1}}\right)^{2}-\left(\mathbb{B C}^{F_{k, n-1}}\right)^{2}=\left[\left(F_{k, n+1}^{2}-F_{k, n-1}^{2}\right)-\left(F_{k, n+2}^{2}-F_{k, n}^{2}\right)-\left(F_{k, n+3}^{2}-F_{k, n+1}^{2}\right)+\left(F_{k, n+4}^{2}-F_{k, n+2}^{2}\right)\right] \\
& +2 i\left[\left(F_{k, n+1} F_{k, n+2}-F_{k, n-1} F_{k, n}\right)-\left(F_{k, n+3} F_{k, n+4}-F_{k, n+1} F_{k, n+2}\right)\right] \\
& +2 j\left[\left(F_{k, n+1} F_{k, n+3}-F_{k, n-1} F_{k, n+1}\right)-\left(F_{k, n+2} F_{k, n+4}-F_{k, n} F_{k, n+2}\right)\right] \\
& +2 i j\left[\left(F_{k, n+1} F_{k, n+4}-F_{k, n-1} F_{k, n+2}\right)+\left(F_{k, n+2} F_{k, n+3}-F_{k, n} F_{k, n+1}\right)\right] \\
& =k\left(F_{k, 2 n}-k F_{k, 2 n+2}-k F_{k, 2 n+4}+k F_{k, 2 n+6}\right) \\
& +2 i\left(k F_{k, 2 n+1}-k F_{k, 2 n+5}\right)+2 j\left(-k^{2} F_{k, 2 n+3}\right)+2 i j\left(2 k F_{k, 2 n+3}\right) \\
& =k\left[\mathbb{B C}^{F_{k, 2 n}}-F_{k, 2 n+2}+k F_{k, 2 n+5}+i\left(F_{k, 2 n+1}-2 F_{k, 2 n+5}\right)\right. \\
& \left.+j\left(-F_{k, 2 n+2}-2 k F_{k, 2 n+3}\right)+i j\left(3 F_{k, 2 n+3}\right)\right] \text {. }
\end{aligned}
$$

(3.5) and (3.6):Proof of equalities can easily be done using Eq.(2.1).

Theorem 3.3. Let $\mathbb{B C}^{F_{k, n}}$ be the bicomplex $k$-Fibonacci quaternion. Then, we have the following identities

$$
\begin{aligned}
& \sum_{s=1}^{n} \mathbb{B} \mathbb{C}^{F_{k, s}}=\frac{1}{k}\left(\mathbb{B} \mathbb{C}^{F_{k, n+1}}+\mathbb{B} \mathbb{C}^{F_{k, n}}-\mathbb{B} \mathbb{C}^{F_{k, 1}}-\mathbb{B C}^{F_{k, 0}}\right), \\
& \sum_{s=1}^{n} \mathbb{B} \mathbb{C}^{F_{k, 2 s-1}}=\frac{1}{k}\left(\mathbb{B} \mathbb{C}^{F_{k, 2 n}}-\mathbb{B} \mathbb{C}^{F_{k, 0}}\right), \\
& \sum_{s=1}^{n} \mathbb{B} \mathbb{C}^{F_{k, 2 s}}=\frac{1}{k}\left(\mathbb{B} \mathbb{C}^{F_{k, 2 n+1}}-\mathbb{B} \mathbb{C}^{F_{k, 1}}\right) .
\end{aligned}
$$

Proof. Proof can be easily done using sums of series following

$\sum_{i=1}^{n} F_{k, i}=\frac{1}{k}\left(F_{k, n+1}+F_{k, n}-1\right), \sum_{i=1}^{n} F_{k, 2 i+1}=\frac{1}{k} F_{k, 2 n+2}$ and $\sum_{i=1}^{n} F_{k, 2 i}=\frac{1}{k}\left(F_{2 n+1}-1\right) \quad$ [1] .

\section{Generating functions and Binet's formula}

In this section, the generating functions and the Binet's formula of the bicomplex $k$-Fibonacci quaternions will be defined.

Theorem 4.1. Let $\mathbb{B C}^{F_{k, n}}$ be the bicomplex $k$-Fibonacci quaternion. For the generating function for these quaternions is as follows:

$$
g_{\mathbb{B} \mathbb{C}^{F_{k, n}}}(t)=\sum_{s=1}^{n} \mathbb{B C}^{F_{k, n}} t^{n}=\frac{\mathbb{B C}^{F_{k, 0}}+\left(\mathbb{B} \mathbb{C}^{F_{k, 1}}-k \mathbb{B C}^{F_{k, 0}}\right) t}{1-k t-t^{2}}
$$

Proof. Using the definition of generating function, we obtain

$$
g_{\mathbb{B} \mathbb{C}_{k, n}^{F_{k, n}}}(t)=\mathbb{B C}^{F_{k, 0}}+\mathbb{B C}^{F_{k, 1}} t+\ldots+\mathbb{B C}^{F_{k, n}} t^{n}+\ldots
$$

Multiplying both sides of Eq.(3.30) and using Eq.(3.7), we have

$$
\left(1-k t-t^{2}\right) g_{\mathbb{B C}^{F_{k, n}}}(t)=\mathbb{B C}^{F_{k, 0}}+\left(\mathbb{B} \mathbb{C}^{F_{k, 1}}-k \mathbb{B}^{F_{k, 0}}\right) t .
$$

Thus, the proof is completed. 
Theorem 4.2. Let $\mathbb{B} \mathbb{C}^{F_{k, n}}$ be the bicomplex $k$-Fibonacci quaternion. For $n \geq 1$, Binet's formula for these quaternions is as follows:

$$
\mathbb{B C}^{F_{k, n}}=\frac{1}{\alpha-\beta}\left(\hat{\alpha} \alpha^{n}-\hat{\beta} \beta^{n}\right)
$$

where

$$
\begin{gathered}
\hat{\alpha}=1+i \alpha+j \alpha^{2}+i j \alpha^{3}, \quad \alpha=\frac{k+\sqrt{k^{2}+4}}{2}, \\
\hat{\beta}=1+i \beta+j \beta^{2}+i j \beta^{3}, \quad \beta=\frac{k-\sqrt{k^{2}+4}}{2} \\
\alpha+\beta=k, \alpha-\beta=\sqrt{k^{2}+4}, \alpha \beta=-1
\end{gathered}
$$

Proof. Using the Binet formula for $k$-Fibonacci number [2], we obtain

$$
\begin{aligned}
\mathbb{B C}^{F_{k, n}} & =F_{k, n}+i F_{k, n+1}+j F_{k, n+2}+i j F_{k, n+3} \\
& =\frac{\alpha^{n}-\beta^{n}}{\sqrt{k^{2}+4}}+i\left(\frac{\alpha^{n+1}-\beta^{n+1}}{\sqrt{k^{2}+4}}\right)+j\left(\frac{\alpha^{n+2}-\beta^{n+2}}{\sqrt{k^{2}+4}}\right)+i j\left(\frac{\alpha^{n+3}-\beta^{n+3}}{\sqrt{k^{2}+4}}\right) \\
& =\frac{\alpha^{n}\left(1+i \alpha+j \alpha^{2}+i j \alpha^{3}\right)-\beta^{n}\left(1+i \beta+j \beta^{2}+i j \beta^{3}\right)}{\sqrt{k^{2}+4}} \\
& =\frac{1}{\sqrt{k^{2}+4}}\left(\hat{\alpha} \alpha^{n}-\hat{\beta} \beta^{n}\right) .
\end{aligned}
$$

where $\hat{\alpha}=1+i \alpha+j \alpha^{2}+i j \alpha^{3}, \hat{\beta}=1+i \beta+j \beta^{2}+i j \beta^{3}$.

\section{Some identities for bicomplex k-Fibonacci quaternions}

\section{Theorem 5.1. Honsberger Identity}

For $n, m \geq 0$ the Honsberger identity for the bicomplex $k$-Fibonacci quaternions is given by

$$
\begin{aligned}
\mathbb{B C}^{F_{k, n}} \mathbb{B} \mathbb{C}^{F_{k, m}}+\mathbb{B C}^{F_{k, n+1}} \mathbb{B C}^{F_{k, m+1}=} & \mathbb{B} \mathbb{C}^{F_{k, n+m+1}}-F_{k, n+m+3}+k F_{k, n+m+6}+i\left(F_{k, n+m+2}-2 F_{k, n+m+6}\right) \\
& +j\left(F_{k, n+m+3}-2 F_{k, n+m+5}\right)+i j\left(3 F_{k, n+m+4}\right) .
\end{aligned}
$$

Proof. (5.1): By the Eq.(3.1) we get,

$$
\begin{aligned}
\mathbb{B C}^{F_{k, n}} \mathbb{B} \mathbb{C}^{F_{k, m}}+\mathbb{B C}^{F_{k, n+1}} \mathbb{B C}^{F_{k, m+1}=} & \left(F_{k, n+m+1}+i F_{k, n+m+2}+j F_{k, n+m+3}+i j F_{k, n+m+4}\right)-F_{k, n+m+3}+k F_{k, n+m+6} \\
& +i\left(F_{k, n+m+2}-2 F_{k, n+m+6}\right)+j\left(F_{k, n+m+3}-2 F_{k, n+m+5}\right)+i j\left(3 F_{k, n+m+4}\right) \\
= & \mathbb{B} \mathbb{C}_{k, n+m+1}-F_{k, n+m+3}+k F_{k, n+m+6}+i\left(F_{k, n+m+2}-2 F_{k, n+m+6}\right) \\
& +j\left(F_{k, n+m+3}-2 F_{k, n+m+3}\right)+i j\left(3 F_{k, n+m+4}\right) .
\end{aligned}
$$

where the identity $F_{k, n} F_{k, m}+F_{k, n+1} F_{k, m+1}=F_{k, n+m+1}$ was used [1].

\section{Theorem 5.2. D'Ocagne's Identity}

For $n, m \geq 0$ the D'Ocagne's identity for the bicomplex $k$-Fibonacci quaternions is given by

$$
\mathbb{B C}^{F_{k, n}} \mathbb{B C}^{F_{k, m+1}}-\mathbb{B C}^{F_{k, n+1}} \mathbb{B C}^{F_{k, m}}=(-1)^{m} F_{k, n-m}\left[2\left(k^{2}+2\right) j+\left(k^{3}+2 k\right) i j\right] .
$$

Proof. (5.2): By the Eq.(3.1) we get,

$$
\begin{aligned}
& \mathbb{B C}^{F_{k, n}} \mathbb{B C}^{F_{k, m+1}}-\mathbb{B C}^{F_{k, n+1}} \mathbb{B C}^{F_{k, m}}=\left[\left(F_{k, n} F_{k, m+1}-F_{k, n+1} F_{k, m}\right)-\left(F_{k, n+1} F_{k, m+2}-F_{k, n+2} F_{k, m+1}\right)\right. \\
& \left.-\left(F_{k, n+2} F_{k, m+3}-F_{k, n+3} F_{k, m+2}\right)+\left(F_{k, n+3} F_{k, m+4}-F_{k, n+4} F_{k, m+3}\right)\right] \\
& +i\left[\left(F_{k, n} F_{k, m+2}-F_{k, n+1} F_{k, m+1}\right)+\left(F_{k, n+1} F_{k, m+1}-F_{k, n+2} F_{k, m}\right)\right. \\
& \left.-\left(F_{k, n+3} F_{k, m+3}-F_{k, n+4} F_{k, m+2}\right)\right] \\
& +j\left[\left(F_{k, n} F_{k, m+3}-F_{k, n+1} F_{k, m+2}\right)+\left(F_{k, n+2} F_{k, m+1}-F_{k, n+3} F_{k, m}\right)\right. \\
& \left.-\left(F_{k, n+1} F_{k, m+4}-F_{k, n+2} F_{k, m+3}\right)-\left(F_{k, n+3} F_{k, m+2}-F_{k, n+4} F_{k, m+1}\right)\right] \\
& +i j\left[\left(F_{k, n} F_{k, m+4}-F_{k, n+1} F_{k, m+3}\right)+\left(F_{k, n+1} F_{k, m+3}-F_{k, n+2} F_{k, m+2}\right)\right. \\
& \left.+\left(F_{k, n+2} F_{k, m+2}-F_{k, n+3} F_{k, m+1}\right)+\left(F_{k, n+3} F_{k, m+1} F_{k, n+4} F_{k, m}\right)\right] \\
& =(-1)^{m} F_{k, n-m}\left[2\left(k^{2}+2\right) j+\left(k^{3}+2 k\right) i j\right] \text {. }
\end{aligned}
$$

where the identity $F_{k, m} F_{k, n+1}-F_{k, m+1} F_{k, n}=(-1)^{n} F_{k, m-n}$ is used [1]. 
Theorem 5.3. Catalan's Identity

Let $\mathbb{B} \mathbb{C}^{F_{k, n+r}}$ be the bicomplex $k$-Fibonacci quaternion. For $n \geq 1$, Catalan's identity for $\mathbb{B C}^{F_{k, n+r}}$ is as follows:

$$
\mathbb{B C}^{F_{k, n+r-1}} \mathbb{B C}^{F_{k, n+r+1}}-\left(\mathbb{B C}^{F_{k, n+r}}\right)^{2}=(-1)^{n+r}\left[2\left(k^{2}+2\right) j+\left(k^{3}+2 k\right) i j\right] .
$$

Proof. (5.3): By using (3.1) we get

$$
\begin{aligned}
\mathbb{B C}^{F_{k, n+r-1}} \mathbb{B}^{F_{k, n+r+1}-\left(\mathbb{B}^{F_{k, n+r}}\right)^{2}=} & \left(F_{k, n+r-1} F_{k, n+r+1}-F_{k, n+r}^{2}\right)-\left(F_{k, n+r} F_{k, n+r+2}-F_{k, n+r+1}^{2}\right) \\
& \left(F_{k, n+r+1} F_{k, n+r+3}-F_{k, n+r+2}^{2}\right)+\left(F_{k, n+r+2} F_{k, n+r+4}-F_{k, n+r+3}^{2}\right) \\
+ & i\left[\left(F_{k, n+r-1} F_{k, n+r+2}\right)-\left(F_{k, n+r} F_{k, n+r+1}\right)-\left(F_{k, n+r+1} F_{k, n+r+4}-F_{k, n+r+2} F_{k, n+r+3}\right)\right. \\
+ & j\left[\left(F_{k, n+r-1} F_{k, n+r+3}-F_{k, n+r} F_{k, n+r+2}\right)-\left(F_{k, n+r} F_{k, n+r+4}-F_{k, n+r+1} F_{k, n+r+3}\right)\right. \\
& \left.+\left(F_{k, n+r+1} F_{k, n+r+1}-F_{k, n+r+2} F_{k, n+r}\right)-\left(F_{k, n+r+2} F_{k, n+r+2}-F_{k, n+r+3} F_{k, n+r+1}\right)\right] \\
+ & i j\left[\left(F_{k, n+r-1} F_{k, n+r+4}-F_{k, n+r} F_{k, n+r+3}\right)\right. \\
& \left.+\left(F_{k, n+r} F_{k, n+r+3}-F_{k, n+r+1} F_{k, n+r+2}\right)+\left(F_{k, n+r+2} F_{k, n+r+1}-F_{k, n+r+3} F_{k, n+r}\right)\right] \\
= & (-1)^{n+r}\left[2\left(k^{2}+2\right) j+\left(k^{3}+2 k\right) i j\right]
\end{aligned}
$$

where the identity of the $k$-Fibonacci numbers $F_{k, n+r-1} F_{k, n+r+1}-F_{k, n+r}^{2}=(-1)^{n+r}$ is used [2]. Furthermore;

$$
\left\{\begin{array}{l}
F_{k, n+r-1} F_{k, n+r+2}+F_{k, n+r} F_{k, n+r+1}=(-1)^{n+r} k, \\
F_{k, n+r-1} F_{k, n+r+3}-F_{k, n+r} F_{k, n+r+2}=(-1)^{n+r}\left(k^{2}+1\right), \\
F_{k, n+r+1} F_{k, n+r+3}-F_{k, n+r} F_{k, n+r+4}=(-1)^{n+r}\left(k^{2}+1\right), \\
F_{k, n+r-1} F_{k, n+r+4}-F_{k, n+r} F_{k, n+r+3}=(-1)^{n+r}\left(k^{3}+2 k\right), \\
F_{k, n+r} F_{k, n+r+3}-F_{k, n+r+1} F_{k, n+r+2}=(-1)^{n+r+1} k, \\
F_{k, n+r+2} F_{k, n+r+1}-F_{k, n+r+3} F_{k, n+r}=(-1)^{n+r} k .
\end{array}\right.
$$

are used.

\section{Theorem 5.4. Cassini's Identity}

Let $\mathbb{B} \mathbb{C}^{F_{k, n}}$ be the bicomplex $k$-Fibonacci quaternion. For $n \geq 1$, Cassini's identity for $\mathbb{B C}^{F_{k, n}}$ is as follows:

$$
\mathbb{B C}^{F_{k, n-1}} \mathbb{B C}^{F_{k, n+1}}-\left(\mathbb{B C}^{F_{k, n}}\right)^{2}=(-1)^{n}\left[2\left(k^{2}+2\right) j+\left(k^{3}+2 k\right) i j\right] .
$$

Proof. (5.4): By using (3.1) we get

$$
\begin{aligned}
& \mathbb{B C}^{F_{k, n-1}} \mathbb{B C}^{F_{k, n+1}}-\left(\mathbb{B C}^{F_{k, n}}\right)^{2}=\left[\left(F_{k, n-1} F_{k, n+1}-F_{k, n}^{2}\right)-\left(F_{k, n} F_{k, n+2}-F_{k, n+1}^{2}\right)\right. \\
& \left.-\left(F_{k, n+1} F_{k, n+3}-F_{k, n+2}^{2}\right)+\left(F_{k, n+2} F_{k, n+4}-F_{k, n+3}^{2}\right)\right] \\
& +i\left[\left(F_{k, n-1} F_{k, n+2}-F_{k, n} F_{k, n+1}\right)-\left(F_{k, n+1} F_{k, n+4}-F_{k, n+2} F_{k, n+3}\right)\right] \\
& +j\left[\left(F_{k, n-1} F_{k, n+3}-F_{k, n} F_{k, n+2}\right)-\left(F_{k, n} F_{k, n+4}-F_{k, n+1} F_{k, n+3}\right)\right. \\
& \left.+\left(F_{k, n+1} F_{k, n+1}-F_{k, n+2} F_{k, n}\right)-\left(F_{k, n+2} F_{k, n+2}-F_{k, n+3} F_{k, n+1}\right)\right] \\
& +i j\left(F_{k, n-1} F_{k, n+4}-F_{k, n} F_{k, n+3}\right) \\
& =(-1)^{n}\left[2\left(k^{2}+2\right) j+\left(k^{3}+2 k\right) i j\right] \text {. }
\end{aligned}
$$

where the identities of the k-Fibonacci numbers $F_{k, n-1} F_{k, n+1}-F_{k, n}^{2}=(-1)^{n}$ [2]. Furthermore;

$$
\left\{\begin{array}{l}
F_{k, n-1} F_{k, n+2}-F_{k, n} F_{k, n+1}=k(-1)^{n} \\
F_{k, n-1} F_{k, n+3}-F_{k, n} F_{k, n+2}=\left(k^{2}+1\right)(-1)^{n}, \\
F_{k, n+1} F_{k, n+3}-F_{k, n} F_{k, n+4}=\left(k^{2}+1\right)(-1)^{n}, \\
F_{k, n-1} F_{k, n+4}-F_{k, n} F_{k, n+3}=\left(k^{3}+2 k\right)(-1)^{n}
\end{array}\right.
$$

are used.

\section{An application of bicomplex $k$-Fibonacci quaternions in tridiagonal matrices}

In this section, we give another method to obtain the $\mathrm{n}$-th term of bicomplex $k$-Fibonacci quaternion sequence as the calculation of a tridiagonal matrix [8]. 
Theorem 6.1. Let $x_{n}$ be any second-order linear sequence, defined recursively as

$$
x_{n+1}=A x_{n}+B x_{n-1}, n \geq 1
$$

with the values $x_{0}=C, x_{1}=D$. Then,for all $n \geq 0$,

$$
x_{n}=\operatorname{det}\left(\begin{array}{ccccccc}
C & D & 0 & 0 & \ldots & 0 & 0 \\
-1 & 0 & B & 0 & \ldots & 0 & 0 \\
0 & -1 & A & B & \ldots & 0 & 0 \\
\vdots & \ddots & \ddots & \ddots & \ddots & \vdots & \vdots \\
0 & 0 & 0 & 0 & \ldots & A & B \\
0 & 0 & 0 & 0 & \ldots & -1 & A
\end{array}\right)
$$

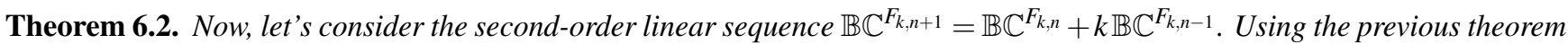
and taking $A=1, B=k, C=\mathbb{B} \mathbb{C}^{F_{k, 0}}, D=\mathbb{B C}^{F_{k, 1}}$ the following determinant was obtained.

$$
\mathbb{B}^{F_{k, n}}=\operatorname{det}\left(\begin{array}{ccccccc}
\mathbb{B}^{C_{k, 0}} & \mathbb{B}^{C^{F, 1}} & 0 & 0 & \ldots & 0 & 0 \\
-1 & 0 & 1 & 0 & \ldots & 0 & 0 \\
0 & -1 & k & 1 & \ldots & 0 & 0 \\
\vdots & \ddots & \ddots & \ddots & \ddots & \vdots & \vdots \\
0 & 0 & 0 & 0 & \ldots & k & 1 \\
0 & 0 & 0 & 0 & \ldots & -1 & k
\end{array}\right)
$$

Proof. For $n \geq 0$, using the above theorem, we have

$$
\begin{aligned}
& \mathbb{B}^{F_{k, 0}}=\left|\mathbb{B C}^{F_{k, 0}} \quad\right|=\mathbb{B} \mathbb{C}^{F_{k, 0}}, \\
& \mathbb{B C}^{F_{k, 1}}=\left|\begin{array}{cc}
\mathbb{B C}^{F_{k, 0}} & \mathbb{B} \mathbb{C}^{F_{k, 1}} \\
-1 & 0
\end{array}\right|=\mathbb{B} \mathbb{C}^{F_{k, 1}}, \\
& \mathbb{B} \mathbb{C}^{F_{k, 2}}=\left|\begin{array}{ccc}
\mathbb{B C}^{F_{k, 0}} & \mathbb{B C}^{F_{k, 1}} & 0 \\
-1 & 0 & 1 \\
0 & -1 & k
\end{array}\right| \\
& =\mathbb{B C}^{F_{k, 0}}\left|\begin{array}{cc}
0 & 1 \\
-1 & k
\end{array}\right|-\mathbb{B} \mathbb{C}^{F_{k, 1}}\left|\begin{array}{cc}
-1 & 1 \\
0 & k
\end{array}\right| \\
& =\mathbb{B} \mathbb{C}^{F_{k, 0}}+k \mathbb{B}^{F_{k, 1}}=\mathbb{B} \mathbb{C}^{F_{k, 2}} \\
& \mathbb{B C}^{F_{k, 3}}=\left|\begin{array}{cccc}
\mathbb{B C}^{F_{k, 0}} & \mathbb{B C}^{F_{k, 1}} & 0 & 0 \\
-1 & 0 & 1 & 0 \\
0 & -1 & k & 1 \\
0 & 0 & -1 & k
\end{array}\right| \\
& =\mathbb{B}^{F_{k, 0}}\left|\begin{array}{ccc}
0 & 1 & 0 \\
-1 & k & 1 \\
0 & -1 & k
\end{array}\right|-\mathbb{B C}^{F_{k, 1}}\left|\begin{array}{ccc}
-1 & 1 & 0 \\
0 & k & 1 \\
0 & -1 & k
\end{array}\right| \\
& =\mathbb{B} \mathbb{C}^{F_{k, 0}(-1)}\left|\begin{array}{cc}
-1 & 1 \\
0 & k
\end{array}\right|-\mathbb{B C}^{F_{k, 1}(-1)}\left|\begin{array}{cc}
k & 1 \\
-1 & k
\end{array}\right| \\
& =k \mathbb{B C}^{F_{k, 0}}+\mathbb{B} \mathbb{C}^{F_{k, 1}}\left(1+k^{2}\right) \\
& =\mathbb{B} \mathbb{C}^{F_{k, 1}}+\left(k^{2} \mathbb{B} \mathbb{C}^{F_{k, 1}}+k \mathbb{B} \mathbb{C}^{F_{k, 0}}\right)=k \mathbb{B C}^{F_{k, 2}}+\mathbb{B} \mathbb{C}^{F_{k, 1}}=\mathbb{B} \mathbb{C}^{F_{k, 3}}
\end{aligned}
$$


In this way, $\mathbb{B} \mathbb{C}^{F_{k, n}} \mathrm{n}$-th term is obtained by calculating the determinant nxn.

\section{Conclusion}

In this paper, a number of new results on bicomplex $k$-Fibonacci quaternions were derived. Furthermore, a different way to find the $\mathrm{n}$-th term of Bicomplex $k$-Fibonacci quaternion sequence was expressed using the determinant of a tridiagonal matrix.

\section{References}

[1] S. Falcon, A. Plaza, On the Fibonacci k-numbers, Chaos Solitons Fractals, 32(5) (2007), 1615-1624.

[2] S. Falcon, A. Plaza, The k-Fibonacci sequence and the pascal 2-triangle, Chaos Solitons Fractals, 33(1) (2007), 38-49.

[3] J. L. Ramirez, Some combinatorial properties of the k-Fibonacci and the k-Lucas quaternions, An. Univ. "Ovidius" Constanta Ser. Mat., 23(2) (2015), 201-212.

[4] C. Segre, Le rappresentazioni reali delle forme complesse e gli ente iper-algebrici, Math. Ann., 40(3) (1892), $413-467$.

[5] G. B. Price, An Introduction to Multicomplex Spaces and Functions, M. Dekker, 1991.

[6] P. Catarino, Bicomplex k-Pell quaternions, Comput. Methods Funct. Theory, 19(1) (2019), 65-76.

[7] F. Aydın, Torunbalc1, Bicomplex Fibonacci quaternions, Chaos Solitons Fractals, 106 (2018), 147-153.

[8] E. K1lıç, D. Tasc1, P. Haukkanen, On the generalized Lucas sequence by Hessenberg matrices, Ars. Comb., 95 (2010), 383-395. 